\section{Expressed emotion as an adaptation to loss*}

\author{
Prospective study in first-episode psychosis \\ PAUL PATTERSON, MAX BIRCHWOOD and RAY COCHRANE
}

The importance of expressed emotion (EE) as a theoretical construct and as a robust predictor of relapse in schizophrenia has been clearly established (Butzlaff \& Hooley, 1998) yet the association of high $\mathrm{EE}$ and increased patient relapse appears to be independent of illness factors (Heikkilla et al, 2002) leaving fundamental questions as to the origin of the emotional responses and direction of influence unanswered. Mechanisms linking EE to family burden and distress are also unclear although several studies implicate carer appraisals as important factors (Barrowclough \& Parle, 1997; Scazufca \& Kuipers, 1999; Raune et al, 2004) and a cross-sectional relationship between EE and family burden has been established (Smith et al, 1993; Scazufca \& Kuipers, 1998) suggesting that EE may be a state characteristic and a 'thermometer of patient-family interactions' (Scazufca \& Kuipers, 1999). It is known that EE is not a stable characteristic, with many changing EE status within 12 months, usually from high to low (Leff et al, 1985; Scazufca \& Kuipers, 1998) and that sub-scales of EE are uncorrelated and associated with different predictor variables (Bentsen et al, 1996, 1998; King \& Dixon, 1996), yet relatively few studies have explored these components of EE for their potential to guide both theory and clinical practice.

Of those that have, some support has been found for an attributional model of EE (Brewin et al, 1991; Barrowclough \& Tarrier, 1992). Higher levels of criticism have been associated with relatives perceiving the cause of patients' behaviours and symptoms as more personal to and controllable by the patient (Brewin et al, 1991; Weisman et al, 1998), more troublesome behaviours in patients (Boye et al, 1999) and failure to engage in productive activity (King et al, 2003), whereas emotional overinvolvement (EOI) was linked to more anxious and depressive symptoms and less aggressive and uncritical behaviour perceived by relatives (Bentsen et al, 1996, 1998).
Barrowclough \& Tarrier (1992) suggest that relatives high in critical relationships (CC) may be displaying 'coercive attempts to restore behaviour' mediated by beliefs of controllability and internal attributions. Emotional overinvolved responses are perhaps less clearly understood but carers who are mothers, single and those who perceive anxiety and depression symptoms in the patients are more likely to be high in EOI (Stubbe et al, 1993; Bentsen et al, 1996), suggesting a transactional process may be operating.

Models of the transactional process between patients and carers explore how each adapts to changes in mental life and behaviour, to the diagnosis and its treatment, and several studies of EE have employed Lazarus \& Folkman's (1994) stress appraisal framework. Birchwood \& Cochrane (1990), Smith et al (1993), Barrowclough \& Parle (1997) and Scazufca \& Kuipers (1999) found carers high in EE perceive greater 'threat', lower control, and report higher distress. One possible form of threat for relatives may be a perceived 'loss' of cherished roles, goals or relationships associated with the unwell family member. Whereas the components of the grieving process have previously been discussed in relation to living with psychosis (Olshansky, 1962; Atkinson, 1994; Davis \& Schultz, 1998) they have seldom been explored quantitatively in response to psychosis (Miller et al, 1990; Atkinson, 1994), or in association with EE (Patterson et al, 2000; Raune et al, 2004).

The present study follows-up a sample of first-episode patients and their relatives to explore the appraisals associated with components of EE and focuses on the following questions concerning the appraisal of loss early in the course of psychosis:

(a) Is loss a feature of carer's initial response to psychosis, particularly in high EE relationships and does this influence the perception of burden?

(b) Do patients appraise loss similarly to carers and are carers and patients' appraisals of loss linked?

(c) Are appraisals of loss in carers connected to depression in the ill relative?

\section{METHOD}

\section{Sampling}

Patients and relatives were selected at the time of the patient's first presentation to services for acute psychosis conforming to 
broad ICD-10 criteria for schizophrenia or related disorder (F20, F22, F23). Patients were considered for inclusion in the study if they had lived or been in regular contact with their relatives for at least 1 of the 3 months prior to admission, had engaged with the health services for the first time for the treatment of psychosis, and had no comorbid major neurological disorders considered to be the primary diagnosis. Patients resident in admission units or managed through home treatment teams of the inner-city area of northern Birmingham and the Gloucester Mental Health Trusts (population 450000) were screened over a 12-month period for a clinical diagnosis of psychosis or schizophrenia and documented symptoms consistent with ICD-10 criteria. Eligible patients and relatives were then approached to participate in the study.

\section{Assessments}

\section{Expressed emotion}

Relatives' EE level was assessed using the Camberwell Family Interview (CFI; Vaughn \& Leff, 1976). A relative was rated as high in EE if they scored 6 or higher in the criticism (CC) sub-scale; 3 or higher in the emotional overinvolvement (EOI) scale or had any score for hostility (H). P.P. was trained in the assessment of $\mathrm{EE}$ to acceptable levels of reliability (overall EE, $\phi=0.84$; CC, $r=0.94 ; \mathrm{H}, \phi=0.85$; EOI, $\phi=0.85)$. A random sample of ten completed CFI interviews from the present study were later rated for reliability and these were also found to have acceptable levels of reliability on overall EE $(\kappa=0.80)$; CC (reliability intraclass coefficient; ICC $=0.74) ; \quad H \quad(\mathrm{ICC}=1.0) ; \quad$ EOI $(\mathrm{ICC}=0.88)$.

\section{Family burden}

The Experience of Caregiving Inventory (ECI; Szmukler et al, 1996) was used to measure salient aspects of caregiving. The ECI is a 66-item questionnaire with ten sub-scales measuring eight negative aspects of caregiving (difficult behaviours; negative symptoms; stigma; problems with services; effects on the family; the need to provide back-up; dependency; loss) and two positive aspects (rewarding personal experiences; good aspects of the relationship with the patient). The negative sub-scales are summed to produce a total 'negative burden' scale. The scale has excellent psychometric properties (Szmukler et al, 1996).

\section{Loss reactions}

Loss was assessed in relatives and patients using the mental illness version of the Texas Revised Inventory of Grief (TRIG; Miller et al, 1990). Originally developed to assess unresolved loss in bereavement (Faschingbauer et al, 1977), it was adapted by Miller $e t$ al to assess loss reactions in relatives of the mentally ill. Items in the TRIG are focused on the 'loss' of aspirations and cherished hopes for the individual and intrusive memories of the individual as he or she used to be. For example, items include: 'I cry when I think how he used to be before the illness', I am preoccupied by thoughts about [he or she] could have been if not for the illness'. The measure therefore comprises a cognitive component, the appraisal of loss, and an emotional component, the distress associated with this appraisal.

The 16 items that comprise the scale are each rated by the individual on a five-point scale from 'completely true' to 'completely false'. The scores are summed. The scale has high internal consistency (Cronbach's $\alpha=0.92$; Miller et al, 1990). For this study we also assessed parallel loss reactions in patients: to do this we adapted each item with a simple change of pronoun (e.g. 'I am preoccupied with the thought of how $I$ could have been if not for the illness').

\section{Depression}

The Calgary Depression Scale for Schizophrenia is an interview-based scale measuring depressed mood, suicidal thinking, self-deprecation and hopelessness. It is in widespread use in schizophrenia, and benefits from minimal overlap with negative symptoms (Addington et al, 1992). In this study we also used it to measure depression in relatives to enable a direct comparison between relatives and patients.

\section{RESULTS}

Fifty patients and 50 key relatives were initially recruited: 6 relatives refused to participate in the follow-up, 2 patients and 1 relative had left the area and a further 2 patients were not in regular contact with their key relative, leaving 39 relative/ patient pairs by follow-up $(78 \%$ of the original sample). The drop-out group did not differ significantly from the remainder on demographic measures or symptom indices.

Among those successfully followed-up, 30 of the relatives were parents $(76.8 \%)$ of which $17(43.5 \%)$ represented singleparent households. There were six partners $(15.3 \%)$ and three siblings $(7.7 \%)$. Relatives' mean age was 43.9 years (s.d.=11.2). The patient sample of 24 males and 15 females with average age at illness onset of 22.8 years (s.d.=5.7) included 28 White $(71.7 \%), 6$ Asian $(15.4 \%)$ and 5 Black or mixed race $(12.8 \%)$ individuals. The majority of patients $(34,85.6 \%)$ returned to live in the family home for the duration of the follow-up period, with the other 5 remaining in regular contact (mean=three times weekly) with their key relative.

\section{Stability and change in expressed emotion}

\section{Expressed emotion at the first episode}

Thirty relatives were high EE $(60 \%)$ and 20 $(40 \%)$ low EE at the first episode. Of the 30 relatives high in EE, 16 were high EOI, 11 high $\mathrm{CC}$ and 11 high $\mathrm{H}$ (with 9 of these high $\mathrm{CC}$ and $\mathrm{H}, 1$ high EOI and CC, 1 high EOI and $\mathrm{H}$ ). The correlation between CC and EOI was $r=-0.12$ (NS). High CC carers had relatives with a longer duration of untreated positive psychotic symptoms prior to first treatment (low $\mathrm{CC}=26.4$ days, high $\quad \mathrm{CC}=85.4$ days, $\quad F(1,37)=5.99$, $P<0.05)$. Expressed emotion status at index and follow-up was significantly but moderately correlated $(r=0.48, P<0.01)$, as was CC $(r=0.55)$ and EOI $(r=0.66)$ but not $\mathrm{H}(r=0.13)$, suggesting considerable stability but also intra-individual change.

There was a considerable change in overall EE status over the follow-up period, with $30.7 \%$ of the relatives obtaining a different EE rating from the initial measure. This was predominantly made up of $42 \%$ of the initially high EE relatives resolving to low EE, whereas only $13 \%$ of the initially low EE relatives resolved to high EE. The individual sub-scales of the CFI showed clear differences in the resolution of EOI and CC in those carers $(n=39)$ followed-up after 9 months. Of 16 carers initially high in EOI, $5(32 \%)$ remained high at follow-up, whereas of 9 carers initially high in CC, $5(56 \%)$ remained high CC. None of those initially high in CC changed to or acquired high EOI at follow-up; this compares with 6 of the 16 
originally high in EOI who were rated high $\mathrm{CC}$ at follow-up and an additional 2 who remained high EOI also rating high in CC at follow-up.

\section{Loss}

\section{Relatives' loss}

The appraisal of loss in key relatives was high at follow-up and similar to levels reported in bereaved individuals (Miller et al, 1990). Atkinson (1994), using the TRIG, found that parents who had lost children through death displayed very high levels of initial perceived loss, but this greatly reduced over time (mean=28.8, s.d.=3.6) and was significantly lower than a matched group of parents who had an offspring with schizophrenia $($ mean $=53.8$, s.d. $=4.1)$. In the present study, there was a significant reduction in carers' loss appraisals from the first episode $(52.5$, s.d. $=11.6)$ to follow-up (45.0, s.d.=10.6), $t=4.7$; $P<0.001$, although relatives' perceived losses at index and follow-up were significantly but moderately correlated $(r=0.55$, $P<0.01$ ), suggesting considerable intraindividual variability over time against this general background of sustained feelings of loss.

\section{Patients' loss}

At the first episode, patients' appraisals of loss was high $(50.3$, s.d. $=11.4)$ and remained high at follow-up $(47.6$, s.d. $=8.7)$, suggesting that the impact of psychosis engenders similar loss reactions in patients and key carers over time. Although at the baseline there were no significant correlations between relatives' and patients' loss (TRIG) scores, by follow-up a significant correlation emerged which was very strong among high EOI relationships (Table 1).

\section{Expressed emotion and loss}

There was no overall difference between high and low EE relatives in loss (TRIG) scores at the first episode; however, high EOI relatives reported significantly higher loss scores than low EOI ones whereas the high CC relatives scored significantly lower than low CC. Direct comparison of those high in EOI only with those high in CC only confirmed that it was the high EOI group who appraised greater loss $(P<0.001)$.
Table I Correlations of patients' with relatives' loss scores at baseline and 9-month follow-up

\begin{tabular}{lcll}
\hline & $r$ (first episode) & $r$ (follow-up) \\
\hline Total sample & $0.01(n=50)$ & $0.425^{* *}$ & $(n=39)$ \\
High EE & $0.05(n=30)$ & $0.40 I$ & $(n=17)$ \\
Low EE & $0.20(n=20)$ & 0.307 & $(n=22)$ \\
High EOI & $0.23(n=20)$ & $0.799 *$ & $(n=7)$ \\
High CC & $-0.33(n=11)$ & 0.108 & $(n=11)$ \\
\hline
\end{tabular}

$E E$, expressed emotion; EOI, emotional overinvolvement; CC, critical relationship.

*Significant at $P=0.05, * *$ Significant at $P=0.01$.

\section{Is there a link between EE and loss?}

Those relatives with initially high levels of EOI which resolved to high CC $(n=8)$ showed a significant reduction in loss from 59.6 (s.d. $=8.5$ ) to 43.4 (s.d. $=10.2$ ), $t=8.2$, $P<0.001$; similarly, those initially high in EOI resolving to low $\mathrm{EE}(n=5)$ also showed a reduction in loss from $57.4($ s.d. $=11.2)$ to 42.2 (s.d.=15.5), $t=4.1, P=0.014$. Those who remained high EOI at follow-up showed no reduction in loss from onset (62.7) to follow-up (58.7). By contrast, the high CC relatives, who started at onset with the lowest loss scores, remained low at follow-up (these relatives either remained high CC or resolved to low EE).

In summary, there was no link with overall EE and loss but very different patterns of loss were observed between high $\mathrm{CC}$ and high EOI relatives:

(a) High EOI relatives had a high appraisal of loss (shared with the patients) and high CC relatives a low appraisal. (b) Loss in high EOI relatives at baseline reduced in parallel with a reduction to low EE or a change to criticism (high $\mathrm{CC})$.

\section{Burden}

\section{At the first episode}

There were no significant differences between high and low EE relatives on subjective burden (ECI). Looking at the subscales, high EOI relatives reported higher burden scores $(84.5$, s.d. $=28)$ than low EOI $(66.4$, s.d. $=17.9), P<0.01$. High CC relatives recorded lower ECI scores (64.1, s.d. $=20.7)$ than low CCs $(76.3$, s.d. $=24.4)$ but this was not significant.

Relatives' loss was correlated with subscales of burden especially the effects on the family and total negative burden and this was stronger at follow-up with the addition of problems with services and dependency.

\section{At follow-up}

High EE relatives perceived significantly higher levels of total negative burden, $F(2,37)=10.85, P=0.002$. This extended to one of the positive scales (positive personal experiences) and suggests that many aspects of the relationship between relatives and patients are more intensely focused on by high EE relatives. Analysis of the EE sub-scales revealed that this effect was entirely due to the high EOI relatives who recorded nearly $50 \%$ higher perceived burden (ECI) scores than low EOI, with the loss sub-scale exercising particular influence (for ECI see Szmukler et al, 1996). Again, no differences emerged between those high $v$. low in CC. Direct comparison between
Table 2 Comparison of high and low EE relatives on ECl burden sub-scales at follow-up

\begin{tabular}{|c|c|c|c|c|c|c|}
\hline & \multicolumn{2}{|c|}{ High EE $(n=22)$} & \multicolumn{2}{|c|}{ Low EE $(n=17)$} & \multirow[t]{2}{*}{$F$} & \multirow[t]{2}{*}{$P$} \\
\hline & Mean & (s.d.) & Mean & (s.d.) & & \\
\hline Total negative behaviours & 85.24 & (30.78) & 57.14 & (22.54) & 10.85 & 0.002 \\
\hline Difficult behaviours & 12.35 & (6.79) & 7.64 & $(4.93)$ & 6.32 & 0.016 \\
\hline Negative symptoms & 9.35 & $(5.24)$ & 7.32 & $(4.64)$ & 1.65 & 0.208 \\
\hline Stigma & 7.12 & $(4.5 I)$ & 4.77 & $(3.59)$ & 3.27 & 0.079 \\
\hline Problems with services & 11.59 & (6.27) & 7.82 & $(4.66)$ & 4.65 & 0.038 \\
\hline Effects on the family & 8.91 & (5.09) & 6.59 & (3.79) & 1.91 & 0.178 \\
\hline Need for back-up & 9.89 & (4.38) & 6.77 & $(5.11)$ & 4.01 & 0.053 \\
\hline Dependency & 10.24 & (4.85) & 6.64 & $(3.51)$ & 7.22 & 0.011 \\
\hline Loss & 13.12 & (4.44) & 8.37 & $(4.96)$ & 9.63 & 0.004 \\
\hline Positive personal experiences & 13.53 & (5.79) & 9.95 & $(5.37)$ & 3.97 & 0.054 \\
\hline
\end{tabular}

$\mathrm{EE}$, expressed emotion; ECI, Experience of Caregiving Inventory. 
those relatives high in EOI only and high in $\mathrm{CC}$ confirms that it is the high EOI group who experience a greater burden $(P<0.01)$.

\section{Loss and burden}

Overall, there was no significant reduction in ECI (burden) scores from onset (73.7, s.d.=25.7) to follow-up $(69.4$, s.d.=29.6), nor did it track any of the movements in the EE sub-scales. Loss was, however, highly correlated with ECI scales 'Total Negative Burden' $(P<0.05)$; 'Effect on the Family' $(P<0.01)$ and 'Loss' $(P<0.01)$ at both onset and follow-up and additionally with 'Dependency' $(P<0.001)$ and Problems with Services $(P<0.01)$ at onset. This suggests that appraisals of burden may reciprocate with the intensity of perceived loss independently of EE levels in carers.

\section{Depression}

\section{Expressed emotion}

There was no relationship between relatives' or patients' depression and EE at onset or follow-up. This was also true of the sub-scales, although high EOI relatives' scores were higher on the Calgary Depression Scale for Schizophrenia (CDS) at onset $(7.8 v .5 .7, \mathrm{NS})$ and at follow-up, where this approached significance, 7.1 (s.d.=4.4) v. 3.9 (s.d.=3.8); $P=0.06$.

\section{Loss and burden}

At follow-up, relatives' depression correlated $(r=0.44 ; P<0.01)$ with loss (TRIG) and with burden $(r=0.43 ; P<0.01)$. In order to determine the principal predictor of depression in relatives at follow-up, a multiple regression analysis was conducted with CDS as a dependent variable and relatives' TRIG, ECI and EOI as independent variables. A step-wise method of entry and removal was used. Only loss was chosen $(R=0.44 ; \beta=0.44, P<0.005)$.

\section{DISCUSSION}

Overall, $28 \%$ of relatives changed EE status during the follow-up period and, in keeping with previous research, this occurred predominantly among the high EE group. At a subsequent relapse, EE may revert back to its original value or there may be further changes - there are no longitudinal data to answer this question. The study replicated the Stirling et al (1993) finding of a number of high EOI relatives changing to or acquiring high
CC, whereas at cross-section the EOI and $\mathrm{CC}$ dimensions were orthogonal. The most striking finding, however, was the pattern of relationships with loss appraisals. Loss was a major feature of the EOI relationship (in relatives and patients) and a reduction in EOI to low EE or a change to high $\mathrm{CC}$ was accompanied by a reduction in loss.

\section{Methodological issues}

The sample employed in the present study is comparable with samples employed by other researchers in this area (e.g. Scazufca $\&$ Kuipers, 1996), particularly first-episode research (Stirling et al, 1993; Raune et al, 2004). The subgroup analyses, particularly those moving from high EOI to high CC, were based on small numbers, although highly significant, and accordingly must be treated as preliminary. The crosssectional comparison of the EOI and CC scales were more robust, being based on larger samples and the relationships with loss were observed at both index and follow-up suggesting that this was not an isolated observation.

The proportion of families from each ethnic group broadly reflected the local demographic profiles, and as such are representative of the areas in which the study was carried out. The findings may not, however, generalise to settings where the ethnic profile is very different. Hashemi \& Cochrane (1999) found different EE profiles in British Asian and White samples, with Muslims more likely than Whites to be rated as high EE. In the present study, only three of the follow-up families were Muslim (one parental, two siblings), with low levels of EE at both time points in two of these and high at onset/low at follow-up in the other. The sample was not epidemiologically based (in common with most other EE studies) which will constrain its generalisability to an unknown degree. Eight out of ten families consented to the follow-up - comparable with similar studies - and no evidence of selective attrition could be found. Overall, the internal and external validity of the study was felt to be sufficiently robust, with the exception of results from some of the subgroup analyses that should be taken as preliminary.

\section{Expressed emotion and loss}

These findings suggest that high EE relationships involving high EOI and CC may have an adaptive function in assisting the relative to respond to the crisis of a family member developing a psychosis. It may be speculated that loss motivates the EOI relationship whose 'over-controlling' qualities may be construed as an attempt to restore the status quo and to mitigate the loss, but what of the inverse relationship between criticism and loss? A clue here may lie in the observation that some EOI relationships evolve to critical ones; suggesting that high CC relationships may be fundamentally underpinned by concern. The accompanying reduction of loss may be adaptive for relatives in easing the distress of an apparently unresolvable situation. Bowlby's (1980) attachment theory demonstrates that coercive criticism is a natural reaction to any perceived loss, having the aim of re-establishing what has been 'lost', such as the pre-existing 'status quo' in the relationship. Rather than suggesting that high EE families are dysfunctional or maladapted, attachment theory suggests that it is normal for a parent or close relative of a patient to express their concerns, or even a reflex 'affectional' response, through criticism. This supports Hooley's view (1986) that high EE may be adaptive, and it is the low EE response which may in fact be unusual when faced with a psychosis. It could be that low EE is reached if there is already experience of mental illness in the family, where coercive measures have been previously tested and rejected, or perhaps in response to a psychosis that has had a long and insidious development, where adaptation within the family has already taken place over many months or years during the prodrome phase. In situations where coercive criticism does not appear to be working, hostility may be expressed which can be conceptualised as the expression of extreme frustration when coercive criticism constantly fails in its objective. The lack of correlation of hostility in relatives from onset to follow-up suggests that this response is less a typical response pattern of relatives and more likely to be reactive to current situational factors (e.g. the frustration at the patient's lack of response to coercion).

\section{Expressed emotion and family burden}

The finding that high EE is associated with higher burden in relatives supports previous findings by Smith et al (1993) and Scazufca 
\& Kuipers (1996, 1998). In the present study we found that burden was a strong feature mainly of the high EOI relationship. It has previously been found that higher burden in relatives is associated with avoidant coping styles and that it is those relatives high in EOI who tend to adopt avoidant coping strategies (Scazufca \& Kuipers, 1999). This, along with the large number of relatives high in EOI at onset, may help to explain the finding that burden in this sample was mainly associated with relatives high in EOI and not those high in CC at both index and follow-up. The levels of distress in relatives may also be different at the first episode than later in the course of illness. Tennakoon et al (2000) found that levels of psychiatric 'caseness' among carers was only $12 \%$ in a first-episode sample, whereas higher rates have been reported among more chronic samples (e.g. $58 \%$ in Budd et al, 1998). The present findings that relatives high in EOI at the first episode tended to be more depressed suggests that EOI and CC warrant further investigation as separate variables. Few studies have examined the separate sub-scales of EE for associations with burden, although Boye et al (1999) did find that patterns of high EOI and high EE at 4.5-month follow-up were associated with increased levels of family burden, in line with the present findings. Also, Barrowclough et al (1996) found that 'self-blaming' beliefs in relatives were associated with increased distress, including EOI relationships. Wynne (1981) has proposed that the two major components of EE - EOI and CC - can be understood as 'special forms of attachment/care-giving that are likely to lead to dysfunctional communicating, problem-solving and intimacy'. Bowlby's work on representational models (Bowlby, 1980) gives some support to the idea that attachment styles may link the development of cognitive biases that in turn may influence the personal interpretation of events such as loss. Examples of this cognitive bias can be seen in more recent literature, such as West et al (1993), who show that components of anxious attachment can differentiate between psychiatric out-patients and the general population in a comparison of clinical and community samples in Canada. Of the components of the attachment scale used, 'feared loss' explained the greatest proportion of the variance, suggesting that the inability to feel secure within relationships is an important correlate of psychiatric disorders and may exacerbate the burden felt by carers.

\section{Duration of untreated psychosis}

The link between longer duration of untreated psychosis (DUP) and high critical relationships (CC) replicates Macmillan et al (1986). The overall duration of DUP in this sample was 63 days (s.d.=96), quite brief compared with many other studies (e.g. Larsen et al, 2000) although this may be due to the present sample living with relatives who are more likely to respond to florid symptoms in patients. This might explain the high rate of EOI seen in this sample, since longer DUP was associated with more criticism.

\section{Implications}

The first major clinical implication is that the early stage of adaptation to psychosis for family members may often involve a process akin to grieving, during which time the relative may experience a high level of subjective burden and is probably less open to, or capable of, integrating psychoeducational interventions. Linszen et al (1996) using Falloon's Behavioural Family Therapy in first-episode psychosis (Falloon et al, 1984), reported that for some relatives this approach interfered with their need to deal with issues related to loss and the 'communication' component of the behavioural training upset carers.

The early phase of psychosis may be a critical period for intervention to prevent high EE, in view of our suggestion that critical responses may be a normal adaptive response to loss involving the reactivation of attachment bonds in the process of 'searching' for the pre-existing relationship (Bowlby, 1980, 1982).

A consideration of the dynamics of grief should play a part in the assessment of carers' needs, particularly at this early stage of adaptation to psychosis. Anger, denial, frustration, a 'search for what has been lost' and later 'acceptance' of the new situation are all recognisable as part of the response to loss.

In summary, although the findings were in line with the hypothesised role for loss, they are also open to other interpretations. One possibility is that 'loss' is a proxy measure for depression, i.e. a mood-linked appraisal, which the correlation with depression (0.44) would support. However, the relationships observed between EE and loss were not mirrored in those between $\mathrm{EE}$ and depression. A second possibility is that EE itself leads relatives to appraise loss in this way, or that loss is intrinsic to the concept of EE. This interpretation is rendered more problematic by the different pattern of links with loss seen within the high EE (EOI/CC) subgroups and their change with time. If the loss appraisal is not, as we believe, causal, we can at least assert that loss is a major feature of these relationships, particularly EOI relationships, and one shared by patients. Some of these possibilities could be examined with a larger study and a longer follow-up period to allow for more detailed observations in the premorbid phase. A truly prospective study of family relationships during the prodromal phase would be the ideal design but is as yet, unfeasible.

Much of the cognitive literature, while placing great emphasis on the importance of appraisal to the interpretation of events and experiences, takes little account of how these appraisals develop. A deeper understanding of loss reactions in relatives of people with psychosis may help to clarify the aetiology of some EE responses from carers attempting to manage the onset of psychosis in family members.

\section{ACKNOWLEDGEMENTS}

We thank Dr Christine Vaughn for her EE training and Dr Liz Dodge for help with reliability rating.

\section{REFERENCES}

Addington, D., Addington, J., Maticka-Tyndale, E., et al (1992) Reliability and validity of a depression rating scale for schizophrenics. Schizophrenia Research, 6, 20I-208.

Atkinson, S. D. (1994) Grieving and loss in parents with a schizophrenic child. American Journal of Psychiatry, I5I, |137-II39.

Barrowclough, C. \& Tarrier, N. (1992) Families of Schizophrenic Patients. Cognitive Behavioural Interventions. London: Chapman and Hall.

Barrowclough, C. \& Parle, M. (1997) Appraisal, psychological adjustment and expressed emotion in relatives of patients suffering from schizophrenia. British Journal of Psychiatry, I7I, 26-30.

Barrowclough, C., Tarrier, N. \& Johnston, M. (1996) Distress, expressed emotion, and attributions in relatives of schizophrenia patients. Schizophrenia Bulletin, 22, 691-702.

Bentsen, H., Boye, B., Munkvold, O. G., et al (1996) Emotional overinvolvement in parents of patients with schizophrenia or related psychosis: Demographic and clinical predictors. British Journal of Psychiatry, 169, 622-630.

Bentsen, H., Notland, T. H., Boye, B., et al (1998) Criticism and hostility in relatives of patients with schizophrenia or related psychoses: demographic and clinical predictors. Acta Psychiatrica Scandinavica, 97, 76-85.

Birchwood, M. \& Cochrane, R. (1990) Families coping with schizophrenia: coping styles, their origins and correlates. Psychological Medicine, 20, 857-865. 
Brewin, C. R., MacCarthy, B., Duda, K., et al (1991) Attribution and expressed emotion in the relatives of patients with schizophrenia. Journal of Abnormal Psychology, 100, 546-554.

Bowlby, J. (1980) Attachment and loss: vol. III. Loss. New York: Basic Books

Bowlby, J. (1982) Attachment and loss: vol. I. Attachment (2nd edn). New York: Basic Books.

Boye, B., Bentsen, H., Notland, T. H., et al (1999) What predicts the course of expressed emotion in relatives of patients with schizophrenia or related psychoses? Social Psychiatry and Psychiatric Epidemiology 34, 35-43.

Budd, R. J., Oles, G. \& Hughes, I. C. (1998) The relationship between coping style and burden in the carers of relatives with schizophrenia. Acta Psychiatrica Scandinavica, 98, 304-309.

Butzlaff, R. L. \& Hooley, J. M. (1998) Expressed emotion and psychiatric relapse: a meta-analysis. Archives of General Psychiatry, 55, 547-552.

Davis, D. J. \& Schultz, C. L (1998) Grief, parenting and schizophrenia. Social Science Medicine, 46, 369-379.

Falloon, I. R., Boyd, J. L. \& McGill, C.W. (1984) Family Care of Schizophrenia. New York: Guilford Press.

Faschingbauer, T. R. (198I) Texas Revised Inventory of Grief Manual. Houston, TX: Honeycomb.

Faschingbauer, T. R., Devaul, R. D. \& Ziscook, S. (1977) Development of the Texas Inventory of Grief. American Journal of Psychiatry, 134, 696-698.

Hashemi, A. \& Cochrane, R. (1999) Expressed emotion and schizophrenia: a review of studies across cultures. International Review of Psychiatry, II, 219-224.

Heikkila, J., Karlsson, H., Taiminen, T., et al (2002) Expressed emotion is not associated with disorder severity in first-episode mental disorder. Psychiatry Research, III, 155-165.

Hooley, J. M. (1986) Expressed emotion and depression: interactions between patients and highversus low-expressed-emotion spouses. Journal of Abnormal Psychology, 95, 237-246.

Hooley, J. M., Orley, J. \& Teasdale, J. D. (1986) Levels of expressed emotion and relapse in depressed patients. British Journal of Psychiatry, 148, 642-647.

King, S. \& Dixon, M. J. (1996) The influence of expressed emotion, family dynamics, and symptom type on the social adjustment of schizophrenic young adults. Archives of General Psychiatry, 53, 1098-1104.

King, S., Ricard, N., Rochon, V., et al (2003) Determinants of expressed emotion in mothers of schizophrenia patients. Psychiatry Research, II7, 211-222

\section{Larsen, T. K., Moe, L. C., Vibe-Hansen, L., et al (2000) Premorbid functioning versus duration of untreated psychosis in I year outcome in first-episode psychosis. Schizophrenia Research, 45, I-9.}

Lazarus, R. S. \& Folkman, S. (1984) Stress, appraisal and coping. New York: Springer.

Leff, J., Kuipers, L., Berkowitz, R., et al (1985) A controlled trial of social intervention in the families of schizophrenic patients: two year follow-up. British Journal of Psychiatry, 146, 594-600.

Linszen, D. H., Dingemans, P. M., Van der Does, A. J. et al (1996) Treatment, expressed emotion and relapse in recent onset schizophrenia. Psychological Medicine, 26 333-342.

Macmillan, J. F., Gold, A., Crow, T. J., et al (1986) Expressed emotion and relapse. British Journal of Psychiatry, 148, 133-143.

\section{CLINICAL IMPLICATIONS}

- Better understanding of loss processes should inform family interventions at first episode.

- Emotional overinvolvement and critical relationships warrant separate exploration for their adaptive as well as maladaptive contributions to family coping.

- The early phase of psychosis may be a critical period for intervention to prevent entrenchment of high expressed emotion.

\section{LIMITATIONS}

Subgroup analyses based on small numbers must be treated as preliminary.

Findings may not generalise to settings where the ethnic profile is very different.

- The sample is not epidemiologically based.

PAUL PATTERSON, PhD, MAX BIRCHWOOD, DSc, School of Psychology, University of Birmingham and Early Intervention Service; RAY COCHRANE, DSc, School of Psychology, University of Birmingham, UK

Correspondence: Dr Paul Patterson, ED:IT, Early Intervention Service, 97 Church Lane, Aston, Birmingham B6 5UG. E-mail: patterpj@bham.ac.uk

Macmillan, F., Crow, T. J., Johnson, A. L., et al (1987) Expressed emotion and relapse in first episodes of schizophrenia. British Journal of Psychiatry, I5I, 320-323.

Miklowitz, D. J., Goldstein, M. J. \& Falloon, I. R. H. (1983) Premorbid and symptomatic characteristics of schizophrenics from families with high and low levels of expressed emotion. Journal of Abnormal Psychology, 92 $359-367$.

Miller, F., Dworkin, J., Ward, M., et al (1990) A preliminary study of unresolved grief in families of seriously mentally ill patients. Hospital and Community Psychiatry, 12, 1321-1325.

Patterson, P., Birchwood, M. \& Cochrane, R. (2000) Preventing the entrenchment of high expressed emotion in first episode psychosis: early developmental attachment pathways. Australian and New Zealand Journal of Psychiatry, 34 (suppl.), SI9I-SI97.

Olshansky, S. (1962) Chronic sorrow: A response to having a mentally defective child. Social Casework, $\mathbf{4 3}$ 190-193.

Raune, D., Kuipers, E. \& Bebbington, P. E. (2004) Expressed emotion at first-episode psychosis: investigating a carer appraisal model. British journal of Psychiatry, 184, 321-326.

Scazufca, M. \& Kuipers, E. (1996) Links between expressed emotion and burden of care in relatives of patients with schizophrenia. British Journal of Psychiatry, 168, 580-587.

Scazufca, M. \& Kuipers, E. (1998) Stability of expressed emotion in relatives of those with schizophrenia and its relationship with burden of care and perception of patients' social functioning. Psychological Medicine, 28, 453-46।.

Scazufca, M. \& Kuipers, E. (1999) Coping strategies in relatives of people with schizophrenia before and after psychiatric admission. British Journal of Psychiatry, 174, 154-158.

Smith, J., Birchwood, M., Cochrane, R., et al (1993) The needs of high and low expressed emotion families: a normative approach. Social Psychiatry and Psychiatric Epidemiology, 28, II-16.

Stirling, J., Tantam, D., Newby, D., et al (1993) Expressed emotion and schizophrenia: the ontogeny of EE during an 18 month follow-up. Psychological Medicine, 23, 77।-778

Stubbe, D. E., Zahner, G. E., Goldstein, M. J., et al (1993) Diagnostic specificity of a brief measure of expressed emotion: a community study of children. Journal of Child Psychology and Psychiatry, 34, 139-154.

Szmukler, G. I., Burgess, P., Herrman, H., et al (1996) Caring for relatives with serious mental illness: the development of the Experience of Caregiving Inventory. Social Psychiatry and Psychiatric Epidemiology, 3I, 137148.

Tennakoon, L., Fannon, D., Doku, V., et al (2000) Experience of caregiving: relatives of people experiencing a first episode of psychosis. British Journal of Psychiatry, 177, 529-533.

Vaughn, C. E. \& Leff, J. (1976) The measurement of expressed emotion in families of psychiatric patients. British Journal of Clinical Psychology, 15, 157-165.

Weisman, A. G., Nuechterlein, K. H., Goldstein, M. J., et al (1998) Expressed emotion, attributions, and schizophrenia symptom dimensions. Journal of Abnormal Psychology, 107, 355-359.

West, M., Rose, M. S. \& Sheldon, A. (1993) Anxious attachment as a determinant of adult psychopathology. Journal of Nervous and Mental Disease, 18I, 422-427.

Wynne, L. C. (198I) Current concepts about schizophrenics and family relationships. Journal of Nervous and Mental Disease, $169,82-89$. 